\title{
Dépistage sérologique de la brucellose porcine et analyse des facteurs de risques dans les élevages à Brazzaville, République du Congo
}

Miassangoumouka ${ }^{1}$ J. P., Amona ${ }^{1}$ I. et H. Banga-Mboko ${ }^{1-2}$. $^{*}$

${ }^{1}$ Institut national de Recherche Agronomique (I.R.A.), BP : 2499, Brazzaville, Congo.

2 Ecole Nationale Supérieure d'Agronomie et de Foresterie, Université Marien NGOUABI, B.P 69 Brazzaville, Congo.

*Auteur correspondant benribangamboko@yahoo.fr, tél +24205 5778025 ou +242 06685 14 76,

Mots-clés : Brucellose, porcs, dépistage, Rose Bengale, facteurs de risques, Brazzaville.

Keywords: Brucellosis, Pigs, RBT, risk factors, Brazzaville.

Publication date 31/10/2019, http://www.m.elewa.org/JAPS

\section{RESUME}

La brucellose est une maladie abortive présente au Congo depuis quelques décennies. La présente étude vérifie l'hypothèse selon laquelle la brucellose sévit dans les élevages porcins de Brazzaville. L'étude avait donc pour objectif d'évaluer la prévalence de l'infection brucellique dans 25 élevages porcins de Brazzaville. Un total de 59 sérums sanguins prélevés sur des échantillons d'animaux non immunisés et en âge de reproduction, a été contrôlé par le test de Rose Bengale. A l'issue du test, sept sérums se sont révélés positifs sur 59 soit une prévalence globale de $11,86 \%$. Une variation du taux d'infection entre les arrondissements, les sexes et les races a été observée. Le taux de prévalence le plus élevé a été noté dans l'arrondissement 9 Madibou (10,17\%). Les verrats ont été plus affectés $(10,63 \%)$ que les truies $(16,66 \%)$. Au niveau des races, les animaux les plus atteints étaient issus des croisements $(20 \%)$ suivi de la race Landrace $(13,63 \%)$, confirmant ainsi l'hypothèse de ce travail. L'infection se perpétue dans les troupeaux à cause des pratiques traditionnelles d'élevage fonctionnant sans suivi zoo sanitaire avec des nombreux échanges de reproducteurs. Pratiques engendrant aussi des risques importants de santé publique. Cette étude suggère un dépistage systématique et régulier de tout le cheptel porcin existant dans tout le pays, y compris à l'importation, suivi de l'abattage des animaux positifs. Il est également impérieux de mener des campagnes de sensibilisation aux populations sur la brucellose et compléter l'étude par le dépistage de l'infection humaine.

Serological testing of porcine brucellosis and analysis of risk factors in pig farming in Brazzaville, Republic of Congo

\begin{abstract}
Brucellosis is an abortive disease most present in Africa and the present study hypothesized the presence of Brucella antibodies in swine in Congo. The aim of the study was to evaluate the prevalence of the brucellosis infection in pig breeding in Brazzaville. Fifty nine 59 blood serums collected from samples of non-immunized animals and at reproduction age, were screened with Rose Bengal Test (RBT). At the end of the test, seven serums were positive with global prevalence of $11.86 \%$, confirming the hypothesis of the presence of the brucellosis in the Congolese pig population. A variation of the rate of infection between
\end{abstract}


districts, sexes and races was observed. The highest prevalence rate was noted in the district 9 Madibou (10.17\%). Boars are more allocated than sows is $16.66 \%$ against $10.63 \%$. The most affected animals come from crosses $(20 \%)$ followed by the Landrace breed $(13.63 \%)$. The infection is dispread in the herds because of the traditional practices of breeding operating without animal health follow-up with many exchanges between breeders. Practices that also generate significant public health risks. Thus, to control swine brucellosis, it is suggested a systematic and regular tracking of all the existing pig herd in all the country, including with the imported pigs, followed by the slaughter of positive animals. It is also imperative to conduct public awareness campaigns on brucellosis and to complete this study by screening for human infection.

\section{INTRODUCTION}

La production porcine, avec un cheptel estimé à 53663 têtes en 2016 (RGA, 2017), occupe une place de choix dans les stratégies de développement de l'élevage au Congo. Cependant, elle ne couvre qu'environ le quart de la consommation nationale de viande de porc, entrainant le recours à des importations pour satisfaire les besoins des populations (FAO, 2009). En effet, cette activité connaît de nombreuses contraintes parmi lesquelles, les pathologies animales persistantes, mal contrôlées ou négligées telle que la brucellose. La brucellose est une maladie infectieuse, contagieuse, commune à de nombreuses espèces animales et a l'Homme. Elle est due à diverses espèces de Brucella (abortus, melitensis, suis, canis...) dont Brucella suis pour les porcins et responsable d'avortements et d'infertilité. C'est l'une des zoonoses les plus répandues à travers le monde (Cutler et al. 2005 ; OIE, 2011). La brucellose freine l'accroissement des cheptels, compromet toute tentative d'amélioration de la productivité des animaux.et réduit l'approvisionnement en viande des populations. Elle représente également une menace sérieuse pour la santé humaine. Le traitement de la brucellose animale par antibiothérapie n'est pas recommandé car non stérilisant et il n'existe aucun vaccin commercial approuvé pour prévenir cette maladie (OIE, 2011 ; Olsen et Tatum, 2017). L'homme est contaminé le plus souvent, par voie cutanéo-muqueuse (contact avec les animaux malades ou leurs produits) ou digestive (consommation des produits animaux infectés) et la maladie se manifeste par de la fièvre ondulante sudoro-algique (Traum, 1914 ; Huddleston, 1929; Cutler et al. 2005, OMS, 2006, Stoffregen 2007, OIE, 2011, Tuon et al., 2017). La brucellose humaine est sousdiagnostiquée en Afrique subsaharienne, sans doute en raison de son tableau clinique parfois confondu à celui d'autres maladies fébriles notamment la fièvre typhoïde et le paludisme. Et pourtant, l'infection a été mise en évidence chez l'homme en Tanzanie (Swai et Schoonman; 2009), à Djibouti (Chantal et al.; 1996) à Ngaoundéré au Cameroun (AwahNdukum et al. ;2018), au Tchad (Schelling et al. ; 2004), en RCA et en Tanzanie (Kunda et al. 2010), au Nigeria (Aworth et al. ; 2013) et au Togo (Dean et al. ; 2013). La maladie animale a été maitrisée dans de nombreux pays développés ayant entraîné une diminution du nombre de cas humains grâce à l'application des mesures de lutte adéquates (Diete-Pérez et al, 2016). Tandis que dans les pays en voie de développement, elle constitue encore de nos jours une source de préoccupation croissante (McDermott et al., 2002 ; Boukary et al., 2014). Les données disponibles énoncent une large distribution de Brucella suis chez les porcs domestiques, les porcs sauvages et chez l'homme en Amérique centrale et du Sud (Meirelles-Bartoli, 2012). L'infection semble endémique dans certaines parties de l'Asie centrale et du sud-est, avec un impact économique majeur en République populaire de Chine en raison des niveaux élevés de production porcine, (Deqiu et al.,2002; Lucero et al., 2008 ; Cvetnic et al.,2009 ; Jiang, 2012). 
En Inde, de nombreuses études ont rapporté l'isolement de Brucella suis (biovar 1) sur des humains infectés par des porcs (Naha et al. 2012 ; Nagalingam et al. 2012). C'est aussi le cas en Indonésie, aux Philippines, à Taiwan, en Polynésie française, en Malaisie, aux Tonga (Praud et al., 2013; Tay et al., 2015; Quance al., 2016) et en Turquie (Kutlu et al, 2016). En Afrique Subsaharienne, il existe de nombreuses études sur la brucellose chez les bovins et les petits ruminants, mais peu d'études ont été réalisées sur la brucellose porcine. Cela est dû aux nombreux tabous sur la viande de porc dans cette région et aux effectifs porcins relativement faibles (Mangen al., 2002 ; McDermott et Arimi, 2002; Schelling et al., 2004 ; Cadmus et al.,2006 ; Boukary et al., 2014).

\section{MATERIEL ET METHODES}

3.1 Zone d'étude : L'étude a été menée à Brazzaville, capitale politique de la République du Congo. Brazzaville est situé au sud du pays sur la rive droite du fleuve Congo. Il compte une population de près de 1.174000 habitants soit $33 \%$ de l'ensemble de la population
$\mathrm{Au}$ Congo, quelques études réalisées sur le dépistage de la brucellose ont révélé l'existence enzootique de la maladie chez les bovins et les petits ruminants (Ngoy et al., 1989 ; Amona et al. , 2016). Les données de l'infection chez les porcins étant rares, l'hypothèse de ce travail repose sur l'existence de la brucellose porcine au Congo. L'objectif général est de garantir la santé publique et la sécurité alimentaire. Ses objectifs spécifiques sont d'évaluer la prévalence de la brucellose par un dépistage sérologique des animaux dans les élevages porcins de Brazzaville et d'identifier les facteurs de risques de transmission de l'infection afin d'élaborer des stratégies de lutte.

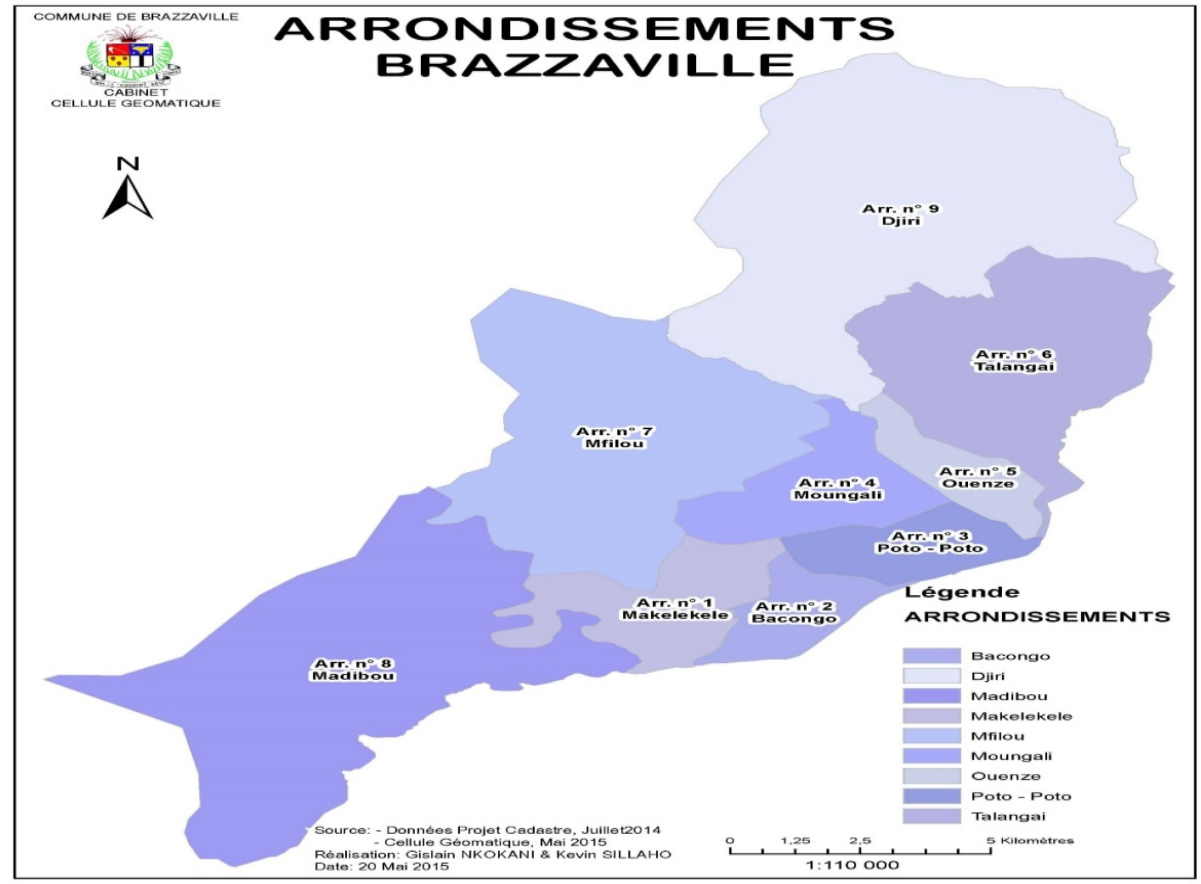

Figure 1 : Carte administrative de Brazzaville (congo-site.com, 2017) 
3.2 Echantillonnage : L'étude a été réalisée sur les animaux en âge de reproduction (mâles et femelles) de la race locale, des races améliorées (Large white, Landrace, Piétrain et Duroc) et des croisés. L'échantillonnage des fermes ayant fait l'objet de cette étude a été constitué à partir du tableau de contingence de Putt et al., (1987) basé sur le rapport: taille de la population, prévalence estimée et probabilité du taux de dépistage. Ainsi, tenant compte de la prévalence de la brucellose à Brucella suis dans la sous-région estimée à 10\% (OMS, 2006) et une probabilité du taux de dépistage de $95 \%$, un échantillon de 25 fermes porcines a été tiré à partir d'une liste de 115 fermes établie par le Ministère de l'Agriculture et de l'Elevage. De ces 25 fermes, 59 animaux ont été retenus et sélectionnés de manière aléatoire (tableau 1).

Tableau 1 : Répartition des élevages porcins dans les arrondissements de Brazzaville

\begin{tabular}{|l|l|l|}
\hline Arrondissements & Nombre total d'élevages & Nombre d'élevages retenus \\
\hline Madibou & 83 & 17 \\
\hline Djiri & 23 & 6 \\
\hline Mfilou & 7 & 2 \\
& & \\
\hline Talangai & 2 & 0 \\
\hline Makélékélé & 0 & 0 \\
\hline Bacongo & 0 & 0 \\
\hline Poto-poto & 0 & 0 \\
\hline Moungali & 0 & 0 \\
\hline Ouenzé & 0 & 0 \\
\hline TOTAL & 115 & 25 \\
\hline
\end{tabular}

\subsection{Enquête dans les fermes porcines:} Parallèlement à la collecte des échantillons biologiques, une enquête a été menée à l'aide de questionnaire simple par un entretien avec le propriétaire de chaque exploitation. Elle a permis d'obtenir des données sur les caractéristiques sociodémographiques, socioéconomiques et culturelles des éleveurs ainsi que les caractéristiques zootechniques et sanitaires des animaux.

\subsection{Prélèvements sanguins : Les}

prélèvements du sang ont été réalisés sur les animaux, le matin entre $8 \mathrm{~h}$ et $11 \mathrm{~h}$. Pendant la contention de l'animal par les porchers, 4 à $5 \mathrm{ml}$ de sang étaient prélevés à l'aide d'une seringue stérile au niveau de la veine auriculaire. Le sang était collecté dans des tubes secs marqués à l'aide d'un codage établi sur la base des renseignements sur l'animal (âge, sexe, race, état physiologique, ferme d'origine). Après prélèvement de sang, les tubes étaient conservés au frais pendant 24 heures pour permettre la décantation du sérum. Le lendemain, les sérums étaient extraits puis acheminés au laboratoire de santé animale de l'Institut national de Recherche Agronomique (IRA) où ont été réalisés les tests.

3.5 Analyse sérologique: Pour le dépistage de la brucellose, les sérums ont été analysés avec le test de Rose Bengale ou Epreuve à l'Antigène Tamponné (EAT) de marque BENGATEST*. C'est une réaction d'agglutination rapide sur lame au moyen d'une suspension de Brucella inactivée par la chaleur et le phénol $(0.5 \%)$, coloré au rose Bengale en milieu acide tamponné (Synbiotics, 2010). Le rose Bengale est l'une des méthodes faciles à mettre en œuvre et largement utilisées dans le dépistage sérologique de la brucellose avec une spécificité de $99.95 \%$ et une sensibilité de 70 $80 \%$ (Bouziri et al., 2011).

3.5.1 Mode opératoire: Sur les lames marquées et étalées sur la table, un volume de $30 \mu \mathrm{l}$ de l'antigène et du sérum à examiner a été prélevé et déposé à l'aide d'une micropipette. L'ensemble antigène-sérum était homogénéisé à 
l'aide de l'embout du cône interchangeable fixé à la micropipette. La lecture a été faite 4 minutes après l'homogénéisation pour un résultat soit négatif (absence d'agglutination ou

\section{$4 \quad$ RESULTATS}

\subsection{Résultats de l'enquête}

4.1.1 Caractéristiques sociodémographiques des éleveurs: L'enquête a révélé que l'élevage de porcs est pratiqué majoritairement par des personnes de classe d'âge compris entre 30 et 40 ans. Le genre masculin représente $88 \%$. Les éleveurs mariés sont minoritaires autour de $20 \%$. Les éleveurs de nationalité congolaise sont les plus nombreux soit environ $96 \%$.

\subsubsection{Caractéristiques socioéconomiques} et culturelles : La plupart des éleveurs enquêtés ont un niveau de scolarisation secondaire et davantage. $95 \%$ ne sont pas fonctionnaires de l'Etat et considèrent l'élevage comme leur profession et le pratiquent depuis plus d'une année comme activité à but commercial.

4.1.3 Caractéristiques zootechniques : Les exploitations possédant les plus gros cheptels se trouvent dans la zone Nord de Brazzaville: le cas de l'arrondissement 8 Djiri. En zone Sud, dans les arrondissements de Madibou et de Mfilou, il y a plus d'élevages mais avec des cheptels réduits (Tableau I). Les races importées notamment la Landrace et la Large white (LW) sont les plus élevées $(75 \%)$ suivies des races Piétrain et Duroc. Mais en général, toutes ces races sont élevées en croisement. Les systèmes d'élevage les plus pratiqués sont de type traditionnel et amélioré.

4.1.4. Caractéristiques sanitaires : Les principales maladies rencontrées dans ces élevages porcins sont souvent saisonnières, Il s'agit de: parasitoses, diarrhées, grippe et rouget. Peu d'éleveurs font appel aux vétérinaires pour les soins. $75 \%$ de cas de mortalités sont souvent remarqués chez les de fixation anticorps-antigène) soit positif (présence d'agglutination ou de fixation anticorps-antigène) (Synbiotics, 2010).

petits à la naissance et surviennent par étouffement ou écrasement par la truie. Le rouget et les endoparasites représentent $15 \%$. Les causes ignorées sont autour de $7 \%$. Selon les éleveurs $95 \%$ des verrats et $80 \%$ des truies présentent des signes d'amaigrissement. Ils ont aussi remarqué $15 \%$ de cas d'avortements répétés chez les femelles et $5 \%$ de stérilité dans les deux sexes. $60 \%$ des éleveurs interrogés procèdent par un emprunt d'un male ou d'une femelle pour la reproduction. Sur la santé des éleveurs enquêtés, aucun signe clinique en rapport avec la brucellose n'a été souligné. Enfin, seuls $5 \%$ des enquêtés ont des connaissances sur la maladie qui sont pour la plupart des anciens étudiants de l'Ecole Nationale Supérieure d'Agronomie et de Foresterie (ENSAF), devenus des éleveurs.

\subsection{Résultats de l'analyse sérologique}

4.2.1 Séroprévalence globale de la brucellose : Après la lecture des résultats du test, les taux de prévalence apparente ont été calculés en utilisant la formule suivante :

Prévalence (\%) = Total animaux positifs au test / Total animaux testés $x 100$

A l'issue du test de Rose Bengale, sept sérums se sont révélés positifs sur 59 échantillons recueillis, soit une prévalence globale de 11,86 $\%$.

4.2.2 Séroprévalence de la brucellose par arrondissement : En fonction de l'arrondissement, le taux de prévalence le plus élevé a été noté dans l'arrondissement 9 Madibou (10,17\%), suivi respectivement de l'arrondissement 8 Djiri $(1,69 \%)$ et de l'arrondissement 7 Mfilou (0\%), situés tous en zone périurbaine (figure 2 ). 


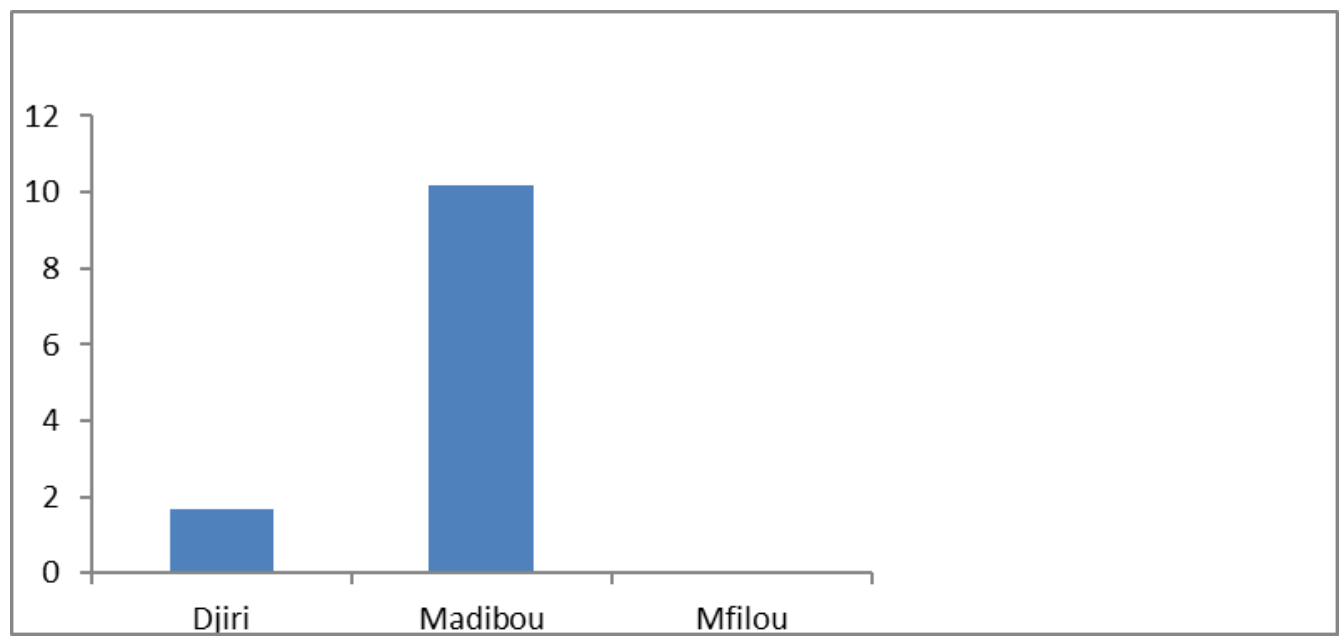

Figure 2 : Prévalence de la brucellose porcine par arrondissement

4.2.3 Prévalence sérologique de la brucellose en fonction du sexe : Selon le sexe, le test de dépistage a révélé un taux de prévalence plus élevé chez les verrats (Tableau 2).
4.2.4 Prévalence de la brucellose en fonction de la race: La réaction positive à l'infection a été plus élevée chez les animaux issus de croisement des races améliorées $(20 \%)$ suivi de la race Landrace $(13,63 \%)$ (Tableau 3)

Tableau 2 : Séroprévalence de la brucellose en fonction du sexe

\begin{tabular}{|l|l|l|l|}
\hline Catégories des animaux/ Sexe & Truies & Verrats & Cheptel \\
\hline Effectif des animaux prélevés & 47 & 12 & 59 \\
\hline Animaux positifs & 5 & 2 & 7 \\
\hline Prévalence (\%) & 10,63 & 16,66 & 11,86 \\
\hline
\end{tabular}

Tableau 3: Prévalence de la brucellose en fonction de la race

\begin{tabular}{|l|l|l|l|l|l|}
\hline Catégories des animaux/ Race & Landrace & LW & Duroc & Croisés & Cheptel \\
\hline Effectif des animaux prélevés & 44 & 9 & 1 & 5 & 59 \\
\hline Animaux positifs & 6 & 0 & 0 & 1 & 7 \\
\hline Prévalence (\%) & 13,63 & 0 & 0 & 20 & 11,86 \\
\hline
\end{tabular}

\section{DISCUSSION}

5.1 Caractéristiques sociodémographiques et économiques des éleveurs: De l'enquête, il s'avère qu'à Brazzaville, les classes d'âge de plus de 30 ans et les hommes sont majoritaires dans cette activité. Cela se justifie d'une part par le choix de l'élevage par ces catégories de personnes matures pour en faire une activité de subsistance ou pour préparer leur retraite, d'autre part l'impératif de disposer des moyens financiers suffisants pour démarrer cet élevage. La plupart des élevages porcins sont situés en zone périurbaine (Djiri, Madibou et Mfilou). Ces élevages sont majoritairement conduits en système traditionnel et amélioré, sans contrôle zoo-sanitaire, avec des échanges de géniteurs (verrats et truies). Ce qui constitue des facteurs de risque importants de transmission de l'infection. De nombreux auteurs de travaux (Faye et al., 2005; Ly, 2007; Swai et Schoonman, 2009 ; Chimana et al., 2010) affirment que dans les milieux périurbains, l'insuffisance d'infrastructures d'assainissement et d'hygiène ainsi que l'absence des services 
vétérinaires contribuent fortement à la dissémination de la brucellose. Peu d'éleveurs ont des connaissances sur la maladie et font appel aux vétérinaires pour les soins. Cependant, le fait que la majorité des éleveurs possède le niveau d'instruction secondaire et davantage, est un atout pour la conduite de l'élevage porcin et dans l'application des bonnes techniques d'alimentation et de suivi sanitaire des animaux. Dans tous les élevages enquêtés, la Landrace est la race dominante pour des raisons d'acclimatation aux conditions du milieu Congolais. Environ $15 \%$ de cas d'avortements répétés chez les femelles ont été notés. Or, la prévalence de la brucellose dans les troupeaux est positivement corrélée avec l'incidence des avortements chez les femelles (McDermott et Arimi, 2002 ; Schelling et al., 2004). Les femelles infectées par la brucellose peuvent excréter des concentrations élevées de l'agent pathogène dans leur lait, les membranes placentaires et les avortons favorisant ainsi la transmission de la maladie aux animaux sains et à l'homme (Corbel, 1988 ; John et al., 2010). Aucun signe clinique en rapport avec la brucellose n'a été noté chez les éleveurs mais les possibilités d'infection restent énormes (Steinmann et al., 2006 ; Kutlu et al, 2016; OIE, 2017). Ces situations de cohabitation homme animal constituent souvent des facteurs de risques potentiels pour la transmission des maladies (Naha et al. 2012 ; Nagalingam et al. 2012 ; Tuon et al., 2017)

\subsection{Séroprévalence de la brucellose}

5.2.1 Séroprévalence globale de la brucellose : Le taux de séroprévalence globale de $11,86 \%$ obtenu dans cette étude est plus élevé que ceux obtenus en 2008 et 2009 au Tchad (4\%) et au Gabon ( $0 \%)$ selon Akakpo et al. (2013) et aussi à Ibadan au Nigeria en 2004 où aucun porc n'a été testé positif sur 200 (94 verrats et 106 truies) (Cadmus et al, 2006). Cependant, ce taux est inférieur à celui obtenu par Bronner et al. (2010) en Polynésie française avec 164 animaux positifs sur 513 animaux testés au Rose Bengale soit une prévalence de $31,96 \%$ et celui de 30\% en Guinée Conakry rapporté par Akakpo et al, (2013), entre 2008 et 2009.

5.2.2 Séroprévalence en fonction du sexe : S'agissant du facteur sexe, les verrats sont plus affectés par l'infection $(16,66 \%)$ que les truies $(10,63 \%)$, conformément aux travaux de Chimana et al (2010) ayant trouvé une séroprévalence brucellique significativement plus élevée chez les mâles que chez les femelles. Inversement, d'autres auteurs (Chantal et Thomas, 1996 ; Traoré et al., 2004 ; Faye et al., 2005) ont rapporté une prévalence sérologique plus importante chez les femelles que chez les mâles, thèse corroborée par Bronner et al, (2010) en Polynésie française dans les élevages de type traditionnel.

5.2.3 Séroprévalence en fonction de la race et des autres facteurs : Les résultats ont aussi montré que les animaux de races importées notamment la Landrace et les croisées sont celles qui présentent plus des cas positifs par rapport aux races locales. Ces résultats concordent avec ceux trouvés par Bronner et al. (2010) et Garin-Bastuji et al., 2010) qui rapportent que les porcs infectés étaient surtout ceux des races améliorées. Les exploitations de type traditionnel pratiquant un élevage sans contrôle zoosanitaire sont les plus exposées. Les risques de transmission de la brucellose y sont généralement plus élevés ou persistants (McDermott et Arimi, 2002 ; Raclozet al, 2013). Ces risques sont encore plus importants dans les zones d'interface entre la faune sauvage et les animaux domestiques (Bengis et al., 2002 ; Muma et al., 2010). En outre, les troupeaux de grande taille sont plus affectés que ceux de petite taille. Ceci s'explique par les contacts rapprochés liés à la densité des animaux. (Racloz et al, 2013).

5.3 Analyses des facteurs de risques: Les risques liés l'infection brucellique sont d'ordres divers notamment liés aux pratiques d'élevage et aux comportements sociaux des individus. La brucellose humaine est considérée comme une maladie professionnelle. Les risques découlent des contacts directs avec les animaux malades ou avec un environnement fortement contaminé. Les catégories 
professionnelles les plus exposées sont : les fermiers et leurs familles, les vétérinaires et agents d'abattoirs, les bouchers, les marchands d'animaux sur pied (Blancou et al., 2005 ; Saegerman et al., 2010 ; Godfroid et al., 2013). Ainsi, Swai et Schoonman (2009) ont trouvé en Tanzanie une prévalence de la brucellose plus élevée chez les agents d'abattoir que chez les autres catégories de travailleurs. De même, Chantal et al. (1996) ont enregistré un taux de prévalence de $6,5 \%$ chez le personnel de l'abattoir de Djibouti. Récemment, les travaux de Awah-Ndukum et al., (2018) ont évoqué une séroprévalence de 5,60\%) parmi le personnel d'abattoir de Ngaoundéré, au Cameroun. Actuellement, l'infection à Brucella suis chez l'homme aux Etats-Unis est principalement associée à l'exposition aux porcs sauvages infectés (sanglier ou autres porcs sauvages) (CDCP, 2009). Chez les éleveurs, la présence des porcins à l'intérieur des concessions ou même dans les cases, la manipulation le plus souvent sans protection des avortons et autres produits excrétés par les animaux lors de parturition sont autant de facteurs de risques de contamination par les Brucella (Godfroid et al., 2013). Les recherches menées par Steinmann et al., (2006) ont montré une séroprévalence de $10,2 \%$ chez les personnes en contact direct avec les animaux et $7,7 \%$ sur les personnes fébriles venues en consultation dans les centres de santé au Mali. Les habitudes alimentaires à risque sont liés à la manipulation et la consommation de produits alimentaires souillés (Godfroid et al., 2003 ; Bonfoh et al., 2003 ; Traoré et al., 2004 ; Kang'Ethe et al., 2007).Dans les milieux urbains et périurbains, Mutanda (1998) a

\section{CONCLUSION ET SUGGESTIONS}

Cette étude avait pour objectif l'évaluation de la prévalence apparente de la brucellose porcine par l'épreuve à l'antigène tamponné (Rose Bengale) dans les élevages de Brazzaville, en République du Congo. Les résultats ont donné une prévalence globale de l'infection animale de 11,86 confirmant la présence de la brucellose dans le cheptel porcin congolais et de suspecter l'existence de cette infection dans la population rapporté une prévalence de 13,3\% chez des patients présentant des symptômes fébriles à l'hôpital de Kampala en Ouganda. Enfin, une étude sur 653 patients fébriles du nord de l'Ethiopie, Brucella abortus a été isolé dans 6,3\% des cas (Yohannes et al., 2013). La brucellose humaine est endémique en Afrique subsaharienne et la séroprévalence rapportée dans certains pays est de 3.8\% au Tchad (Schelling et al. 2004), 3.3\% en RCA, $7.7 \%$ et en Tanzanie (Kunda et al. 2010), 24.1\% au Nigeria (Aworth et al. 2013) et $1-5.6 \%$ au sein des éleveurs traditionnels(Fulani) et $0-1.6 \%$ parmi les nonéleveurs au Togo (Dean et al. 2013). En Afrique, l'absence de frontières naturelles entre les pays voisins, le cas du Congo Brazzaville, avec d'importants courants d'échanges commerciaux sont des facteurs favorisant l'extension de l'infection. Ces courants d'échanges sont souvent responsables de l'introduction des maladies. En Polynésie Française, les études ont montré que la présence de la brucellose était due aux voyageurs partant dans des localités infectées et rapportant la maladie à leur retour. Ainsi, en 2010, le nombre d'élevages connus comme infectés par la brucellose représentait près de 80\% des élevages testés. (Garin-Bastuji.et al., 2010). Enfin, au Congo, le taux de séroprévalence brucellique chez les humains n'est pas connu mais celui de $11,86 \%$ obtenu dans cette étude constitue un risque de santé publique surtout pour la population exposée dans les situations de cohabitation hommeanimal. Ainsi l'hypothèse selon laquelle la brucellose porcine est présente au Congo, a été vérifiée dans la présente étude.

humaine exposée. Cette étude a permis également de constater une variation de la séroprévalence entre les arrondissements, les sexes et les races. L'infection se perpétue dans les troupeaux à cause des pratiques traditionnelles d'élevage présentant des facteurs de risque car fonctionnant sans suivi sanitaire avec des nombreux échanges de reproducteurs. Ainsi, la lutte contre cette pathologie 
zoonotique s'avère une nécessité incontournable afin de diminuer sa prévalence. Un dépistage régulier de tout le cheptel porcin devrait être effectué suivi de l'abattage systématique des animaux positifs (principe du dépistage/abattage) en y associant un contrôle strict des animaux importés. Le fait que le Congo soit entouré par des pays où des foyers sont régulièrement déclarés, le peuplement et le repeuplement des élevages devraient se faire en dehors de ces pays. Le dépistage de l'infection humaine contribuerait aussi à lutter contre cette zoonose. Ainsi, La mise en place d'un cadre multisectoriel impliquant les médecins, les vétérinaires et toutes les parties prenantes œuvrant dans la santé publique et animale dans

\section{REMERCIEMENTS}

Ce travail a été financé par l'Institut National de Recherche Agronomique. Les auteurs remercient Ir Miénandi Nkodia Maguy de le contexte d'une approche «One Health» est recommandée. Le développement de l'insémination artificielle devrait également favoriser le contrôle de la brucellose porcine au Congo Brazzaville. La lutte contre la maladie passe également par la sensibilisation des éleveurs aux problèmes de la brucellose incluant les consignes de prudence en présence de certains signes cliniques et l'obligation de faire appel aux services vétérinaires en cas de suspicion. Il serait également impérieux de compléter cette étude par le dépistage de l'infection chez les porcs des autres localités et par la détermination de la prévalence réelle de la maladie et la caractérisation des souches circulantes de Brucella spp. .

l'Ecole Nationale Supérieure d'Agronomie et de Foresterie, Université Marien NGOUABI, pour son assistance technique.

\section{REFERENCES BIBLIOGRAPHIQUES}

Akakpo A.J. et Ndour A.P.N. 2013. La brucellose bovine en Afrique de l'ouest et du centre : état des lieux, $R A S P A$, $11: 23-28$.

Amona I, Miassangoumouka J.P., BangaMboko H., Adzona P.P., Rabeson F. A.et Ikolakoumou J. 2016. Dépistage sérologique de la brucellose bovine par l'épreuve à l'antigène tamponné (EAT) et l'ELISA dans un centre de multiplication et de métayage bovin en république du Congo-Brazzaville. J. Anim., Plant Sci., 27(3): 4315-4329.

Awah-Ndukum,J., Moctar Mouliom Mouiche M.,Kouonmo-Ngnoyum, L. Houli Bayang, N. ,Rodrigue Simonet Namegni Poueme, Kouamo J., Ngu-Ngwa,V., 1 Assana E., Kameni Feussom, J.M et Pagnah Zoli A.2018. Seroprevalence and risk factors of brucellosis among slaughtered indigenous cattle, abattoir personnel and pregnant women in Ngaoundéré, Cameroon. BMC Infectious Diseases, 18:611.
Aworh MK, Okolocha E, Kwaga J, Fasina F, Lazarus D, Suleman I, Poggensee G, Nguku P. et Nsubuga P. 2013. Human brucellosis: seroprevalence and associated exposure factors among abattoir workers in Abuja, Nigeria. Pan Afr Med J.; 16:103.

Blancou B., Chomel B., Belotto A., Melesen F.X., 2005. Emerging or re-merging bacterial zoonoses: factors of emergence, surveillance and control. Vet. Res. 36: 507-522.

Bonfoh B., Fane A., Steiman P., Hetzel M., Traoré A.N., Traoré M., Sembé C.F., Alfaroukh I.O., Nicoleti., Akakpo J.A.et Zinsstag J. 2003. Qualité microbiologique du lait et produits laitiers vendus au Mali et leur implication sur la santé publique. Etud. Rech. Sabéliennes, 8: 19-27.

Boukary A.R., Saegerman C., AdehossiE., Matthys F., Vias G.F., Yenikoye A.et Thys E. 2014. La brucellose en Afrique subsaharienne. Ann. Méd. Vét., 158 : 3956. 
Bouziri D., Benyamina K.et Goucem R. 2011. Etude comparative de la valeur de détectabilité de l'EAT et de la technique ELISA dans le diagnostic de la Brucellose.Rev Méd. Econ, 10 : 2-7.

Bronner A.et Garin-Bastuji B. 2010. Bilan de la surveillance de la brucellose porcine en 2009 : détection de foyers sporadiques en élevage plein air, Bull. Epidémiol. Santé Anim. Alim., 40:32-34.

CDCP (Centers for Disease Control et Prevention). 2009. Brucella suis infection associated with feral swine hunting three states, 2007-2008. Morb Mortal Wkely Rep. ; 58(22):618-621.

Chantal J., Bessiere M.H., Le Guenno B., MagnavalJ.F.et Dorchies P., 1996. Serologic screening of certain zoonoses in the abattoir personnel in Djibouti.Bull. Soc. Pathol. Exot., 89:353357.

Chimana H.M., Muma J.B.,Samui K.L., Hangombe B.M., Munyeme M., Matope G., Phiri A.M., GodfroidJ., Skjerve E.et Tryland M., 2010. A comparative study of seroprevalence of brucellosis in commercial and small-scale mixed dairybeef cattle enterprises of Lusaka province and Chibombo district,Zambia. Trop. Anim. Health Prod., 42: 1541-1545.

Congo-site. République du Congo. 2017. Site officiel: www.congo-site.com.

Corbel M.1988. Brucellosis. In: Laing J., Brinley Morgan W.J.et Wagner W.C. (Eds), Fertility and infertility in veterinary practice, Baillière Tindall, Londres, 190221.

Cutler S.J., Whatmore A.M., Commander A.J. 2005. Brucellosis--new aspects of an old disease J. Appl Microbiol. 98:1270-1281.

Cvetnić Z, Špičić S, Tončić J, D. Majnarić Benić , D. Albert D, Thiébaud M et Garin-Bastuji B.2009.. Brucella suis infection in domestic pigs and wild boar in Croatia. Rev Sci Tech; 28 (3):10571067.
Dean AS, Bonfoh B, Kulo AE, Boukaya GA, Amidou M, Hattendorf J, Pilo P et Schelling E. 2013. Epidemiology of brucellosis and Q fever in linked human and animal populations in northern Togo. PLoS One. 2013; 8(8):e71501.

Deqiu S, Donglou X et Jiming Y. 2002. Epidemiology and control of brucellosis in China. Vet Microbiol., 90(1-4), 165182.

Delafosse A., Goutard F.et Thébaud E., 2002. Épidémiologie de la tuberculose et de la brucellose des bovins en zone périurbaine d'Abéché, Tchad. Rev. Élev. Méd. Vét. Pays Trop., 55 (1) : 5-13.

Diete-Pérez L, Frankena K, Blasco JM, Munoz PM et de Jong MC.2016. Efficacy of antibiotic treatment and test-based culling strategies for eradicating brucellosis in commercial swine herds. Prev Vet Med.; 126:105-110

El-Anasry E.H., Mohammed B.A., Hamad A.R.et Karom A.G., 2001 Brucellosis among animals human contacts in eastern Sudan. Saudi Med. J., 22:577-579.

FAO. 2009. Etats de lieux et cartographie de la filière bovine au Congo. 2009. Schéma directeur pour le développement des filières d'élevages, FAO, Rome, 67.

Faye B., Castel V., Lesnoff M., Rutabinda D.et Dhalwaj. 2005. Tuberculosis and brucellosis prevalence survey on dairy cattle in Mbarara milk basin (Uganda). Prev. Vet. Med., 67: 267-281.

Garin-Bastuji B., Benoit D., Hautier J.P., Campos V. 2010, La brucellose porcine en Polynésie française. Bulletin épidémiologique, santé animale et alimentation, $43: 35-38$.

Godfroid J., Al Dahouk S., Pappase G., RothfF.,Matopeg G., Mumah J.,Marcotty T., Pfeiffer D.et Skjerveke, 2013. A “One Health"surveillance and control of brucellosisi developing countries:moving away from improvisation.Comp. Immunol. Microbiol.Infect. Dis., 36: 241-248. 
Huddleston IF. 1929. The differentiation of the species Genus Brucella. Bull Mich Agric Exp Stn 100.

Jiang $H$, Chen $H$, Chen JD, Tian GZ, Zhao HY, Piao DR et Cui BY.. 2012. Genetic comparison of Brucella suis biovar 3 in clinical cases in China. Vet Microbiol. 160(3-4):546-548.

John K., Fitzpatrick J.,French N., Kazwala R.,Kambarage D., Mfinanga S.G., Macmillan. A.et Cleaveland S., 2010. Quantifying Risk Factors for Human Brucellosis in Rural Northern 53 Tanzania. PLoS ONE, 5, e9968, doi: 10.1371/journal. pone.0009968.

Kang'ethe E.K., EkuttanC.E., Kimani V.N.et Kiragu M. W. 2007. Investigations into the prevalence of bovine brucellosis and the risk factors that predispose humans to infection among urban dairy and non-dairy farming households in Dagoretti division, Nairobi,Kenya. East Afr. Med. J., 84: 96-99.

Kunda J, Fitzpatrick J, French N, Kazwala R, Kambarage D, Mfinanga GS, MacMillan A et Cleaveland S. 2010. Quantifying risk factors for human brucellosis in rural northern Tanzania. PLoS One. 2010; 5(4):e9968.

Kutlu M, Cevahir N, Erdenlig-Gurbilek S, Akalin S, Ucar M. et Sayin-Kutlu S. 2016. The first report of Brucella suis isolation in human in Turkey. I Infect Public Health. Epub 2016 Mar 3.

Lucero NE, Ayala SM, Escobar GI, Jacob NR. 2008. Brucella isolated from humans and animals in Latin America from 1968 to 2006. Epidemiol Infect.,136(4):496$503)$.

Ly C. 2007. Santé animale et pauvreté en Afrique. In : Mbaye A.A.,Roland-Holst D., Otte J. (Eds), Agriculture, élevage et pauvreté en Afrique de l'Ouest. CREA: Rome, 7, 71-85.

Mangen, M.-J., Otte, J., Pfeiffer, D.et Chilonda P.,2002. Bovine brucellosis in SubSaharan Africa: Estimation of seroprevalence and impact on meat and milk off take potential, livestock information and policy branch, AGAL, FAO, 53.

McDermott JJ, Arimi SM. 2002. Brucellosis in sub-Saharan Africa: epidemiology, control and impact. Vet Microbiol. ; 90(1-4):111-134.

Meirelles-Bartoli R.B, Mathias LA, Samartino L.E. 2012. Brucellosis due to Brucella suis in a swine herd associated with a human clinical case in the state of Sao Paulo, Brazil. Trop Anim Health Prod:; 44(7):1575-1579.

Muma J.B., Lund A., Siamudaala V. M .,Munang'Andu H.M.,Munyeme M., Matope G.,Nielsen K., Djonne B.,Godfroid J., Tryland M.et Skjerve E., 2010.SerosurveyofBrucella spp. infection in the Kafue lechwe (Kobus leche kafuensis) of the Kafue flats in Zambia. J. Wildl. Dis., 46:1063-1069.

Mutanda L.N.1998. Selected laboratory tests in febrile patients in Kampala, Uganda. East Afr. Med.J., 75: 68-72.

Nagalingam $\quad \mathrm{M}^{1}$, Shome $\quad \mathrm{R}$, Balamurugan V, Shome BR, NarayanaRao K, Vivekananda, Isloor S. et Prabhudas K.. 2012. Molecular typing of Brucella species isolates from livestock and human. Trop Anim Health Prod.; 44(1):59).

Naha K, Dasari S, Pandit V, Shubha Seshadri. 2012. A rare case of seronegative culture-proven infection with Brucella suis. Australas Med J. 5(7):340-343.

Ngoy J.J.et Kiafuta D., 1989. État sanitaire du bétail dans un ranch bovin en République populaire du Congo (ranch de Louila).Bull. Anim. Health. Prod. Afr.,37: 333-336.

OIE., 2011. La brucellose. Fiche d'information générale sur les maladies, $6 \mathrm{p}$.

Olsen SC et Tatum FM. 2017. Swine brucellosis: current perspectives. Veterinary Medicine: Research and Reports, Volume 8, 1-12. 
OMS. 2006. Brucellosis in humans and animals; World Health Organisation. WHO/CDS/EPR/2006, 86p.

Praud A, Gimenez O, Zanella G, 2013. Evaluation of five serologic tests for the diagnosis of porcine brucellosis in French Polynesia. Trop Anim Health Prod:;45(4):931-933.

Putt S.N.H., Shaw A.N.H., Woods A.J., Tyler L.et James A.D. 1987. Approche épidémiologique de l'étude des maladies. In: épidémiologie et économie en Afrique centrale, CIPA, 29-53.

Racloz V., Schelling E.,Chitnis N. et Roth F.,Zinsstag J. 2013. Persistence of brucellosis in pastoral systems.Rev. Sci. Tech. Off. Int. Epiq., 32: 61-70.

RGA [Recensement général de l'agriculture] Congo. 2017, Synthèse des analyses des résultats définitifs du Recensement général de l'agriculture (RGA 20142017) en République du Congo, Volume IV, 107p

Saegerman C., Berkvens D., Godfroid J., WalravensK., 2013. Bovine brucellosis. Bayesian estimation of true prevalence,sensitivity and specificity of rose bengal test and indirect ELISA for diagnosis of bovine brucellosis in Ivory Coast. Vet. J.,195:114-120.

Schelling E., Diguimaye C., Daoud S., Nicolet J.et Zinsstag J., 2004. Séroprévalences des maladies zoonotiques chez les pasteurs nomades et leurs animaux dans le Chari-Baguirmidu Tchad. Med. Trop., $64: 474-477$.

Steinmann P., Bonfoh B.et Traoré M., 2006. Séroprévalence de la brucellose et les facteurs de risque pour la séroconversion des personnes fébriles dans les centres de santé urbains au Mali. $R A S P A, 4$ : 3-4.

Stoffregen WC, Olsen SC et Wheeler J. 2007. Diagnostic characterization of a feral swine herd enzootically infected with Brucella. J Vet Diagn Invest. 2007;19(3):227-237.

Swai E.S., Schoonman L. 2009. Human brucellosis: seroprevalence and risk factors related to high risk occupational groups in Tanga Municipality, Tanzania. Zoonoses Public Health, 56 : 183-187.

Synbiotics. 2010. Notice d'usage Bengatest.Synbiotics Europe, France, 2.

Traum J. 1914. Annual report to the chief of the Bureau of Animal Industry, year ending June 30, 1914. USDA Bureau of Animal Industry; 1914:30.

Traoré A., Hamidou H.T., Balé B., David W.R., Nongasida Y.et Moumouni S. 2004. Prévalence globale des pathologies majeures liées à la production laitière bovine en système d'élevage intraurbain à Hamdallaye (Ouagadougou). Biotechnol. Agron. Soc. Environ., 8: 3-8.

Quance C., Robbe-Austerman S., Stuber T., Brignole T., DeBess E.E, Boyd L., Master B.L., Rebekah T, Draper J, Humphrey S, et Erdman M. 2016. Identification of source of B. suis infection in human by using wholegenome sequencing, United States and Tonga. Emerg Infect Dis.; 22(1):79-82.

Yohannes M., DegefuH., Tolosa T., BelihuK., Cutler R. 2013. Cutler S. Brucellosis in Ethiopia. Afr. J.Microbiol. Res., 7: 11501157. 